\title{
Using a multidimensional unfolding approach to assess multiple sclerosis patient preferences for disease-modifying therapy: a pilot study
}

This article was published in the following Dove Press journal:

Patient Preference and Adherence

29 May 2017

Number of times this article has been viewed

\author{
Angel Perez Sempere' \\ Vanesa Vera-Lopez ${ }^{2}$ \\ Juana Gimenez-Martinez' \\ Elena Ruiz-Beato ${ }^{3}$ \\ Jesús Cuervo ${ }^{4}$ \\ Jorge Maurino 5 \\ 'Department of Neurology, Hospital \\ General Universitario de Alicante, \\ Alicante, ${ }^{2}$ Department of Statistics, \\ University of Salamanca, Salamanca, \\ ${ }^{3}$ Health Economics and Outcomes \\ Research Unit, Roche Farma SA, \\ Madrid, ${ }^{4} \mathrm{Health}$ Economics and \\ Outcomes Research, Oviedo, ${ }^{5}$ Medical \\ Department, Roche Farma SA, \\ Madrid, Spain
}

Purpose: Multidimensional unfolding is a multivariate method to assess preferences using a small sample size, a geometric model locating individuals and alternatives as points in a joint space. The objective was to evaluate relapsing-remitting multiple sclerosis (RRMS) patient preferences toward key disease-modifying therapy (DMT) attributes using multidimensional unfolding.

Patients and methods: A cross-sectional pilot study in RRMS patients was conducted. Drug attributes included relapse prevention, disease progression prevention, side-effect risk and route and schedule of administration. Assessment of preferences was performed through a five-card game. Patients were asked to value attributes from 1 (most preferred) to 5 (least preferred).

Results: A total of 37 patients were included; the mean age was 38.6 years, and $78.4 \%$ were female. Disease progression prevention was the most important factor (51.4\%), followed by relapse prevention (40.5\%). The frequency of administration had the lowest preference rating for $56.8 \%$ of patients. Finally, $19.6 \%$ valued the side-effect risk attribute as having low/very low importance.

Conclusion: Patients' perspective for DMT attributes may provide valuable information to facilitate shared decision-making. Efficacy attributes were the most important drug characteristics for RRMS patients. Multidimensional unfolding seems to be a feasible approach to assess preferences in multiple sclerosis patients. Further elicitation studies using multidimensional unfolding with other stated choice methods are necessary to confirm these findings

Keywords: multiple sclerosis, multidimensional unfolding, patient preferences, diseasemodifying therapy, decision-making

\section{Introduction}

Multiple sclerosis (MS) is a chronic autoimmune, inflammatory neurological disease of the central nervous system. ${ }^{1}$ Despite no curative treatment for MS, the past 2 decades have seen 12 different disease-modifying therapies (DMTs) being approved for relapsing-remitting MS (RRMS). ${ }^{2}$ However, treatment decisions are becoming more challenging due to the more diverse risk-benefit spectrum associated with new DMTs. ${ }^{3}$

Incorporating patient characteristics and preferences is crucial to achieve personalized MS treatment. ${ }^{4}$ Patients are increasingly demanding a more active role in their medical care. Thus, in the management of MS, it is important to involve patients in shared decision-making processes related to their treatment initiation or switching due to the unique risk-benefit profile associated with each DMT. ${ }^{5}$
Medical Department, Roche Farma SA,

Eucalipto 33, Madrid 28016, Spain

Tel +34 9I 3248173

Email jorge.maurino@roche.com 
In the real-world setting, patients evaluate a range of features to make decisions. The analysis of preferences can be used to further improve the knowledge of which treatment attributes are considered the most valuable by patients. ${ }^{6,7}$ There are different methodologies for assessing patient preferences for treatment alternatives based on the description of main attributes. The conjoint analysis, a multivariate technique originally used to estimate the value that people give to the attributes that define products and services, is commonly utilized in health care research to determine the relative weight of significant attributes that comprehensively define the conceptual framework underlying preferences for a given treatment. ${ }^{8}$ Multidimensional unfolding is a method that allows both subjects and stimuli to be presented graphically in a common plane according to their preferences. ${ }^{9-11}$ This methodology would permit the exploration of patient preferences for different attributes with a small sample size while providing robust results, thus overcoming the difficulty of large sample sizes needed in classical methods such as conjoint analysis. ${ }^{9}$

The main objective of this pilot study was to assess patient preferences for a range of DMT attributes for RRMS using a multidimensional unfolding approach.

\section{Patients and methods}

A noninterventional, cross-sectional pilot study was conducted in adult patients with a diagnosis of RRMS (2010 McDonald criteria), an Expanded Disability Status Scale (EDSS) score of 0-6.0, and who were receiving a DMT..$^{12,13}$ The study was performed in the MS clinic of a university hospital, and patients were consecutively included between September and October 2015. The study was conducted according to the principles of the Declaration of Helsinki and approved by the Institutional Review Board of the Hospital General Universitario de Alicante (Alicante, Spain). All patients provided written informed consent to participate in this study.

Sociodemographic and clinical characteristics of the sample and patient-reported questionnaires were also collected. The EDSS was used to measure their level of disability. Health-related quality of life (HRQoL) was assessed using the EuroQol Five Dimensions Questionnaire (EQ-5D). ${ }^{14}$ Patient perceptions of how well clinician performance fitted the shared decision-making process was evaluated with the nine-item Shared Decision-Making Questionnaire (SDMQ-9), a patient-reported outcome tool ranging from 0 to 100 with higher scores indicating a greater extent of shared decision-making. ${ }^{15}$
DMT attributes were developed through a review of current clinical trial literature and advanced clinical expertise. ${ }^{2,16}$ A total of five attributes were finally defined taking into account the most important characteristics of available DMTs: relapse prevention, disease progression prevention, side-effect risk, route of administration and frequency of administration. The elicitation survey was performed through a five-card game. Patients were asked to rank the described attributes from 1 (most preferred) to 5 (least preferred).

Patient preferences were analyzed using multidimensional unfolding, a simple geometric and exploratory method in which each drug attribute is ordered according to patient preferences and converted into Euclidean distances in a dimensional space. The attributes and patients are therefore represented together, thus allowing the distance of each individual to the attribute to be directly related to their preference. Under this approach, each patient will remain closer to his/ her most preferred attribute. ${ }^{11,17}$

Descriptive analysis of the sample and the multidimensional unfolding were conducted using the SPSS V20.0 and PREFSCAL.

\section{Results}

A total of 37 patients were included in the study. The mean age was $38.6 \pm 2.0$ years, and $78.4 \%$ were female. The mean time since MS diagnosis was $8.8 \pm 1.1$ years, and median EDSS score was $2.0 \pm 1.5$. The most common DMTs were first-line injectable agents ( $43.2 \%$ of patients), followed by dimethyl fumarate $(24.3 \%)$, and natalizumab (16.2\%). The main sociodemographic and clinical characteristics of the sample are presented in Table 1.

The HRQoL outcome indicated a moderate-to-high self-assessed health status with a mean visual analog scale (VAS) score of 76.7 \pm 15.5 . Most patients considered that their involvement in their treatment decision-making was adequate (mean SDMQ-9 total score of 81.6 \pm 3.2 ; Figure 1).

Figure 2 shows a graphical representation of preferences by means of the distance between patients' stated preferences: white dots represent the responses of each participant with their corresponding numerical identification and the attributes considered. Most patients were concentrated around disease progression prevention (51.4\%) representing the most relevant DMT domain. More specifically, the relative importance of each attribute is shown in Figure 3: disease progression prevention was followed by relapse prevention (40.5\%). The frequency of administration had the lowest preference for $56.8 \%$ of the patients, while $21.6 \%$ of them valued the sideeffect risk attribute as having low to very low importance. 
Table I Sociodemographic and clinical characteristics of the sample

\begin{tabular}{ll}
\hline Characteristics & $\mathbf{N}=\mathbf{3 7}$ \\
\hline Age, years, mean (SD) & $38.6(2.0)$ \\
Female, $\mathrm{n}(\%)$ & $29(78.4)$ \\
Level of education, $\mathrm{n}(\%)$ & \\
$\quad$ Primary school & $10(27.0)$ \\
Secondary school & $13(35.1)$ \\
University & $14(37.8)$ \\
Employment status, $\mathrm{n}(\%)$ & \\
Employed & $18(48.6)$ \\
Unemployed & $9(24.3)$ \\
Retired (due to MS) & $6(16.2)$ \\
Unpaid activity & $4(10.8)$ \\
Time since MS diagnosis, years, mean (SD) & $8.5(1.1)$ \\
Number of relapses since diagnosis, mean (SD) & $2.17(0.5)$ \\
Number of relapses in the last I2 months, $\mathrm{n}(\%)$ & \\
0 & $33(89.2)$ \\
I & $2(5.4)$ \\
2 & $2(5.4)$ \\
Time since last relapse, months, mean (SD) & $60.8(7.2)$ \\
EDSS score, median (SD) & $2.0(1.5)$ \\
SDMQ-9, mean (SD) & $81.6(19.8)$ \\
Current DMT treatment, $\mathrm{n}$ (\%) & \\
Injectable first-line DMTs & $16(43.2)$ \\
Dimethyl fumarate & $9(24.3)$ \\
Fingolimod & $4(10.8)$ \\
Time on current DMT, years, mean (SD) & $4.0(0.6)$ \\
Number of prior DMTs, $\mathrm{n}(\%)$ & $14(37.8)$ \\
0 & $14(37.8)$ \\
I & $9(24.3)$ \\
$\geq 2$ & \\
\hline
\end{tabular}

Abbreviations: DMT, disease-modifying therapy; EDSS, Expanded Disability Status Scale; MS, multiple sclerosis; SD, standard deviation; SDMQ-9, nine-item Shared Decision-Making Questionnaire.

\section{Discussion}

A recent systematic review of qualitative and quantitative research to understand the experiences of health care professional (HCP)-patient interactions in MS shows that patients are given limited opportunity and choice for decisions about involvement in their treatment and care. ${ }^{18}$ Involving MS patients in the decision-making process is crucial for selecting the treatment that best suits the patients' objectives, preferences and lifestyles. ${ }^{5,19,20}$ Our pilot study using multidimensional unfolding suggests that efficacy is the most important drug attribute for almost all participating patients. In contrast, the frequency and route of administration appear to be the least important characteristics for DMT selection.

There are different methodologies for assessing patient preferences for treatment alternatives. The conjoint analysis is the most commonly utilized in health care research. ${ }^{8}$ The multidimensional unfolding is an alternative approach consisting in a geometric model that isolates individuals and alternatives as pinpoints in a joint space. To our knowledge, this is the first study applying multidimensional unfolding in the field of MS care.

Several studies that identified MS DMT patient preferences have been published in the last years. ${ }^{21-26}$ However, limitations such as non-representative patient sampling, incomplete exploration of drug attribute spectrum and the application of heterogeneous analytical methods have limited generalizability. The findings from the current study are aligned with recent publications using conjoint analysis but requiring higher samples. In a sample of 651 patients from the USA, the most important DMT factor was identified as a delay in years to disability progression, followed by the risk of progressive multifocal leukoencephalopathy. ${ }^{21}$ Poulos et $\mathrm{al}^{22,23}$ conducted two studies identifying the number of years until MS symptoms get worse as being the most important attribute, followed by flu-like symptoms, frequency of injections per month and number of relapses in the following years. In another study, 55 patients with RRMS completed a survey showing the highest preference for DMT that would improve MS symptoms and prevent progression, but not on relapse prevention. ${ }^{24}$ Furthermore, a study using latent class modeling conducted in Canada, with a sample of 189 patients with RRMS and primary progressive MS, concluded that the most important drug attribute was the avoidance of serious adverse effects..$^{25}$ In summary, symptom improvement and prevention of disease progression were the most sought-after benefits related to a DMT preference. However, Wilson et $\mathrm{al}^{16}$ identified that patients receiving the first-generation DMTs showed more aversion to fatal safety risk than those receiving the high-efficacy DMTs, fingolimod or natalizumab. The ability of natalizumab-treated patients to assume therapy-associated risks and the factors involved in such risk acceptance was assessed in a study by Tur et al. ${ }^{26}$ The authors defined risk acceptance as a multifactorial phenomenon which is partly explained by an adaptive process involving the perception of MS as a more severe disease.

\section{Limitations}

Our pilot study has several limitations. First, it is a singlecenter study. Second, the assessment of DMT attributes in terms of comprehension and importance by the target population in a previous phase before conducting the study was not performed. Third, the population was composed of a sample of clinically stable patients (a mean of 5 years since the last relapse) with mild disability, mostly employed and with a good self-perception of their health status. Therefore, the results may not be generalizable to less stable subjects. Finally, we did not include psychiatric comorbidity and 


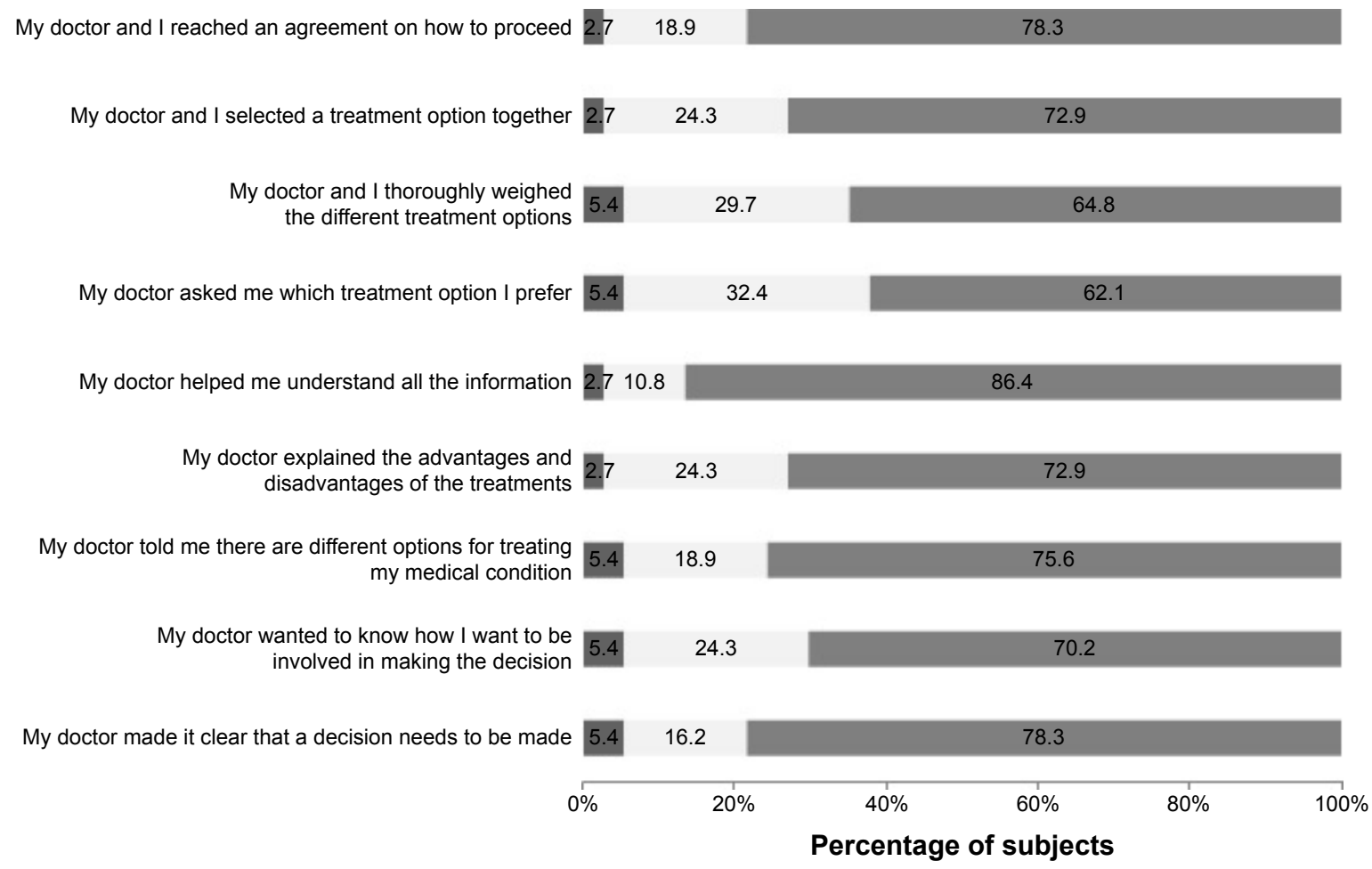

$\square$ Completely/somewhat disagree $\square$ Somewhat agree $\square$ Completely/strongly agree

Figure I Results of the SDMQ-9.

Abbreviation: SDMQ-9, nine-item Shared Decision-Making Questionnaire.

cognitive impairment as factors that might be associated with patient preferences.

\section{Conclusion}

Understanding which MS treatment characteristics are meaningful to patients may help to improve a better $\mathrm{HCP}-$ patient

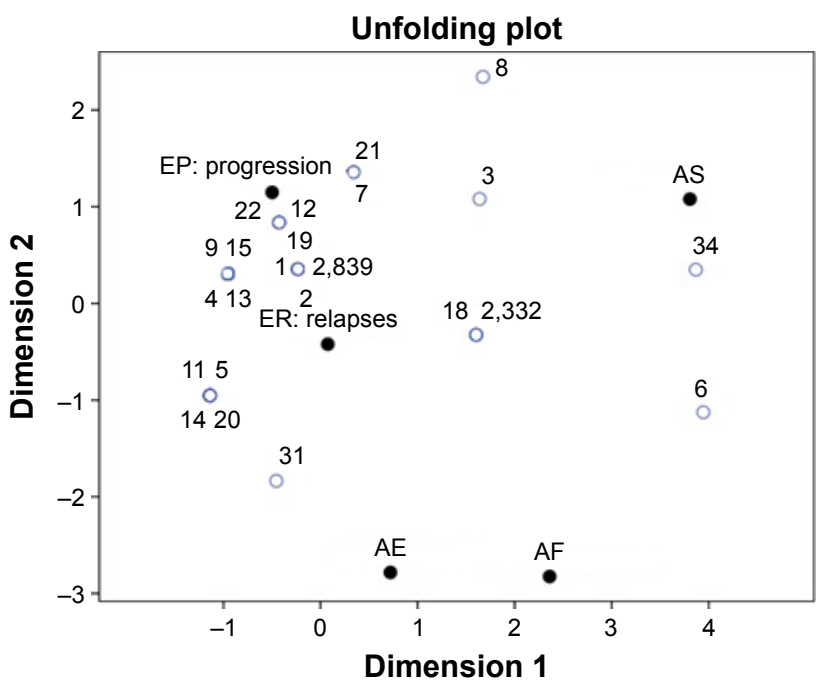

Figure 2 Joint plot for preference rankings of DMT attributes.

Abbreviations: $A E$, adverse events; $A F$, administration form; $A S$, administration schedule; DMT, disease-modifying therapy; EP, efficacy - progression; ER, efficacy progression. interaction and shared decision-making. Multidimensional unfolding seems to be a feasible method with clear advantages. This graphical summary for each individual patient is a clear representation of the real clinical setting that may facilitate the patients' choice among the wide variety of available DMTs. Besides, this approach may permit the exploration of patient preferences for different drug attributes with smaller sample sizes than classical techniques. However, further studies combining multidimensional unfolding with

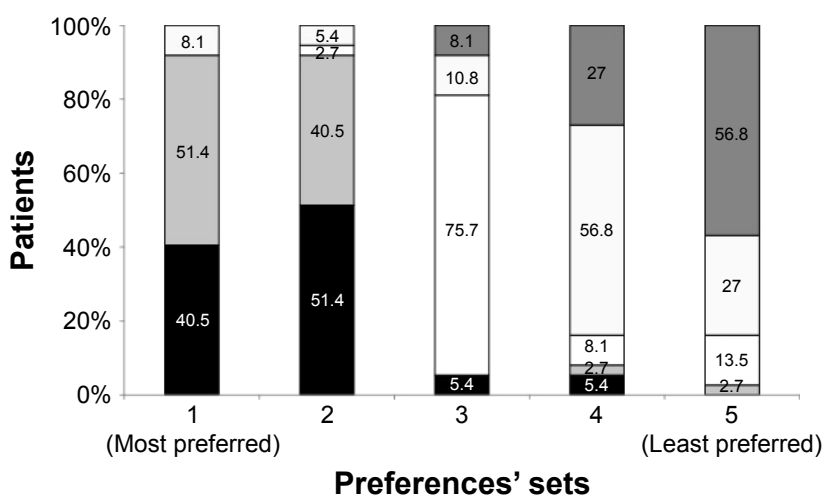

- Prevention of relapses a Prevention of progression $\square$ Adverse events $\square$ Route of administration $\square$ Schedule of administration

Figure 3 Relapsing-remitting patient preferences. 
conjoint analysis are necessary to elucidate whether this analytical approach could be a valid and an efficient alternative to evaluate patient preferences toward treatments.

\section{Disclosure}

The study was funded by Roche Farma SA, Spain. Elena Ruiz-Beato and Jorge Maurino are employees of Roche Farma SA. The other authors report no conflicts of interest in this work.

\section{References}

1. Compston A, Coles A. Multiple sclerosis. Lancet. 2008;372(9648): 1502-1517.

2. Torkildsen O, Myhr KM, Bo L. Disease-modifying treatments for multiple sclerosis-a review of approved medications. Eur J Neurol. 2016;23(suppl 1):18-27.

3. Sempere AP, Gimenez-Martinez J. Safety considerations when choosing the appropriate treatment for patients with multiple sclerosis. Expert Opin Drug Saf. 2014;13(10):1287-1289.

4. Gafson A, Craner MJ, Mattthews PM. Personalised medicine for multiple sclerosis care. Mult Scler. 2017;23(3):362-369.

5. Colligan E, Metzler A, Tiryaki E. Shared decision-making in multiple sclerosis: a review. Mult Scler. 2017;23(2):185-190.

6. Ryan M, Scott DA, Reeves C, et al. Eliciting public preferences for healthcare: a systematic review of techniques. Health Technol Assess. 2001;5(5):1-186.

7. Fried TR. Shared decision making-finding the sweet spot. $N$ Engl $J$ Med. 2016;374(2):104-106.

8. Bridges HP, Huber AB, Marshall D, et al. Conjoint analysis applications in health: a checklist of the ISPOR good research practices for conjoint analysis task force. Value Health. 2011;14(4):403-413.

9. De Leeuw J. Multidimensional unfolding. In: Everitt BS, Howell DC, editors. Encyclopedia of Statistics in Behavioral Science. New York: Wiley; 2005:1289-1294.

10. Zaborski A. Geometrical presentation of preferences by using unfolding models. Acta Universitatis Lodziensis Folia Oeconomica. 2010; 235:365-373.

11. De Sarbo WS, Kim S. A review of the major multidimensional scaling models for the analysis of preference-dominance data in marketing. In: Mouthinho L, Huarng K, editors. Quantitative Modelling in Marketing and Management. London: World Scientific Press; 2013:3-25.

12. Polman $\mathrm{CH}$, Reingold SC, Banwell B, et al. Diagnostic criteria for multiple sclerosis: 2010 revisions to the McDonald criteria. Ann Neurol. 2011;69(2):292-302.
13. Kurtzke JF. Rating neurologic impairment in multiple sclerosis: an expanded disability status scale (EDSS). Neurology. 1983;33(11): $1444-1452$.

14. Badia X, Roset M, Monserrat S, Herdman M, Segura A. The Spanish version of EuroQoL: a description and its applications. Med Clin (Barcelona). 1999;112(suppl 1):79-85.

15. De las Cuevas C, Perestelo-Perez L, Rivero-Santana A, CebollaMartí A, Scholl I, Härter M. Validation of the Spanish version of the 9-item Shared Decision-Making Questionnaire. Health Expect. 2015; 18(6):2143-2153.

16. Wilson L, Loucks A, Bui C, et al. Patient-centered decision making: use of conjoint analysis to determine risk-benefit trade-offs for preference sensitive treatment choices. J Neurol Sci. 2014;344(1-2):80-87.

17. Busing FMTA, Groenen PJF, Heiser WJ. Avoiding degeneracy in multidimensional unfolding by penalizing on the coefficient of variation. Psychometrika. 2005;70(1):49-76.

18. Soundy A, Roskell C, Adams R, et al. Understanding health care professional-patient interactions in multiple sclerosis: a systematic review and thematic synthesis. Open J Ther Rehabil. 2016;4:187-217.

19. Heesen C, Solari A, Giordano A, Kasper J, Köpke S. Decisions on multiple sclerosis immunotherapy: new treatment complexities urge patient engagement. J Neurol Sci. 2011;306(1-2):192-197.

20. Lorefice L, Mura G, Coni G, et al. What do multiple sclerosis patients and their caregivers perceive as unmet needs? BMC Neurol. 2013; 13:177.

21. Johnson FR, Van Houtven G, Ozdemir S, Hass S, White J, Miller DW. Multiple sclerosis patients' benefit-risk preferences: serious adverse events risks versus treatment efficacy. $J$ Neurol. 2009;256(4):554-562.

22. Poulos C, Kinter E, Yang JC, Bridges JF, Posner J, Reder AT. Patient preferences for injectable treatments for multiple sclerosis in the United States: a Discrete-choice experiment. Patient. 2016;9(2):171-180.

23. Poulos C, Kinter E, Yang JC, et al. A discrete-choice experiment to determine patient preferences for injectable multiple sclerosis treatments in Germany. Ther Adv Neurol Disord. 2016;9(2):95-104.

24. Wilson L, Loucks A, Gipson G, et al. Patient preferences for attributes of multiple sclerosis disease-modifying therapies development and results of a ratings-based conjoint analysis. Int J MS Care. 2015; 17(2):74-82.

25. Lynd LD, Traboulsee A, Marra CA, et al. Quantitative analysis of multiple sclerosis patients' preferences for drug treatments: a best-worst scaling study. Ther Adv Neurol Disord. 2016;9(4):287-296.

26. Tur C, Tintoré M, Vidal-Jordana Á, et al. Risk acceptance in multiple sclerosis patients on natalizumab treatment. PLoS One. 2013 8(12):e82796.
Patient Preference and Adherence

\section{Publish your work in this journal}

Patient Preference and Adherence is an international, peer-reviewed, open access journal that focuses on the growing importance of patient preference and adherence throughout the therapeutic continuum. Patient satisfaction, acceptability, quality of life, compliance, persistence and their role in developing new therapeutic modalities and compounds to optimize

\section{Dovepress}

clinical outcomes for existing disease states are major areas of interest for the journal. This journal has been accepted for indexing on PubMed Central. The manuscript management system is completely online and includes a very quick and fair peer-review system, which is all easy to use. Visit http://www. dovepress.com/testimonials.php to read real quotes from published authors. 